\title{
VII Index des verbes selon leur fréquence-type
}

Exemple: le verbe écrire se trouve dans 73 groupes syntaxiques différents, c'est-à-dire, il se combine avec 73 adjectifs-adverbes différents

\begin{tabular}{|c|c|c|c|c|c|c|}
\hline écrire & 73 & demander & 10 & croire & 6 & siffler \\
\hline parler & 68 & descendre & 10 & défendre & 6 & sortir \\
\hline vivre & 50 & mourir & 10 & dresser & 6 & soutenir \\
\hline sonner & 47 & raisonner & 10 & lier & 6 & traire \\
\hline faire & 45 & tricoter & 10 & partir & 6 & trotter \\
\hline voir & 40 & battre & 9 & pédaler & 6 & agir \\
\hline jouer & 39 & férir & 9 & piquer & 6 & asseoir \\
\hline manger & 39 & mener & 9 & planter & 6 & avoir \\
\hline aller & 38 & pleurer & 9 & pleuvoir & 6 & bouter \\
\hline habiller & 36 & souffler & 9 & prier & 6 & clamer \\
\hline tenir & 32 & tirer & 9 & rêver & 6 & crier \\
\hline chanter & 29 & causer & 8 & toucher & 6 & croître \\
\hline marcher & 29 & commencer & 8 & vendre & 6 & danser \\
\hline dire & 28 & compter & 8 & voter & 6 & déclarer \\
\hline penser & 28 & filer & 8 & abattre & 5 & envoyer \\
\hline rire & 25 & jeter & 8 & arriver & 5 & errer \\
\hline boire & 21 & lancer & 8 & cogner & 5 & flairer \\
\hline peindre & 20 & louer & 8 & conduire & 5 & friser \\
\hline regarder & 20 & passer & 8 & connaître & 5 & ganter \\
\hline répondre & 20 & payer & 8 & construire & 5 & geler \\
\hline venir & 20 & rayonner & 8 & continuer & 5 & gémir \\
\hline tomber & 19 & soupirer & 8 & donner & 5 & hausser \\
\hline monter & 18 & sourire & 8 & éclairer & 5 & jaillir \\
\hline acheter & 17 & tailler & 8 & embrasser & 5 & maintenir \\
\hline rouler & 17 & travailler & 8 & entrer & 5 & mentir \\
\hline sentir & 17 & appeler & 7 & étendre & 5 & meubler \\
\hline couler & 15 & arrêter & 7 & fâcher & 5 & moucher \\
\hline porter & 15 & avaler & 7 & flamber & 5 & naître \\
\hline prendre & 15 & brûler & 7 & fleurer & 5 & nommer \\
\hline dessiner & 14 & coiffer & 7 & gâcher & 5 & nouer \\
\hline entendre & 14 & couper & 7 & gagner & 5 & pêcher \\
\hline aimer & 13 & cuisiner & 7 & glisser & 5 & peser \\
\hline mettre & 13 & juger & 7 & gronder & 5 & poindre \\
\hline vêtir & 13 & respirer & 7 & jurer & 5 & promettre \\
\hline chevaucher & 12 & saluer & 7 & lire & 5 & regretter \\
\hline dormir & 12 & savoir & 7 & loger & 5 & saillir \\
\hline frapper & 12 & servir & 7 & luire & 5 & sangloter \\
\hline courir & 11 & taper & 7 & menacer & 5 & sauter \\
\hline cracher & 11 & trancher & 7 & pendre & 5 & semer \\
\hline résonner & 11 & baiser & 6 & plaindre & 5 & serrer \\
\hline tourner & 11 & claquer & 6 & pousser & 5 & tendre \\
\hline chausser & 10 & coucher & 6 & prononcer & 5 & tinter \\
\hline conter & 10 & coûter & 6 & retenir & 5 & tousser \\
\hline
\end{tabular}




\begin{tabular}{|c|c|c|c|c|c|c|}
\hline tuer & 4 & prouver & 3 & conseiller & 2 & lâcher \\
\hline vibrer & 4 & publier & 3 & contrôler & 2 & litronner \\
\hline viser & 4 & rager & 3 & copier & 2 & maudire \\
\hline aboyer & 3 & rapporter & 3 & couronner & 2 & mesurer \\
\hline accuser & 3 & remuer & 3 & crever & 2 & moquer \\
\hline affirmer & 3 & renifler & 3 & crocher & 2 & moudre \\
\hline assaillir & 3 & reprendre & 3 & cuire & 2 & mouiller \\
\hline atourner & 3 & reprocher & 3 & demeurer & 2 & nier \\
\hline attacher & 3 & rester & 3 & dénoncer & 2 & noter \\
\hline bruire & 3 & retourner & 3 & désirer & 2 & oloir \\
\hline chauffer & 3 & revenir & 3 & détester & 2 & opérer \\
\hline chercher & 3 & rompre & 3 & disputer & 2 & ouïr \\
\hline chier & 3 & secouer & 3 & écarter & 2 & ouvrer \\
\hline choir & 3 & songer & 3 & écouter & 2 & pardonner \\
\hline confesser & 3 & tondre & 3 & élever & 2 & parier \\
\hline corner & 3 & valoir & 3 & emmener & 2 & parvenir \\
\hline coudre & 3 & absorber & 2 & empoigner & 2 & percer \\
\hline deviser & 3 & accélérer & 2 & enfler & 2 & perdre \\
\hline diriger & 3 & adorer & 2 & enfoncer & 2 & piocher \\
\hline douter & 3 & agiter & 2 & engager & 2 & placer \\
\hline éclater & 3 & ajouter & 2 & ennuyer & 2 & pondre \\
\hline écrier & 3 & allonger & 2 & enrager & 2 & proférer \\
\hline élancer & 3 & allumer & 2 & enseigner & 2 & proposer \\
\hline émouvoir & 3 & amuser & 2 & esgarder & 2 & puer \\
\hline endormir & 3 & annoncer & 2 & étamper & 2 & raconter \\
\hline estimer & 3 & applaudir & 2 & éteindre & 2 & randonner \\
\hline hacher & 3 & apprendre & 2 & étrangler & 2 & raser \\
\hline ignorer & 3 & approcher & 2 & étreindre & 2 & rayer \\
\hline incliner & 3 & ardre & 2 & étudier & 2 & recevoir \\
\hline jaser & 3 & armer & 2 & finir & 2 & rechercher \\
\hline labourer & 3 & assener & 2 & fourmiller & 2 & réciter \\
\hline lacer & 3 & atterrir & 2 & fuir & 2 & réclamer \\
\hline laver & 3 & baisser & 2 & fumer & 2 & recorder \\
\hline miser & 3 & bâtir & 2 & garder & 2 & récriminer \\
\hline mordre & 3 & bêcher & 2 & gérer & 2 & redouter \\
\hline murmurer & 3 & boutonner & 2 & gésir & 2 & reflamboyer \\
\hline navrer & 3 & briller & 2 & grever & 2 & refuser \\
\hline odorer & 3 & briser & 2 & guerroyer & 2 & régler \\
\hline organiser & 3 & buquer & 2 & guier & 2 & rendre \\
\hline ouvrir & 3 & calculer & 2 & harmoniser & 2 & répéter \\
\hline péter & 3 & caqueter & 2 & heurter & 2 & répliquer \\
\hline pincer & 3 & changer & 2 & hucher & 2 & rincer \\
\hline pisser & 3 & chantonner & 2 & hurler & 2 & seoir \\
\hline poser & 3 & chasser & 2 & interroger & 2 & signifier \\
\hline posséder & 3 & cheminer & 2 & issir & 2 & soulever \\
\hline préparer & 3 & coloïer & 2 & joindre & 2 & souquer \\
\hline presser & 3 & combattre & 2 & joster & 2 & suer \\
\hline priser & 3 & commenter & 2 & juter & 2 & supplier \\
\hline produire & 3 & comprendre & 2 & kiffer & 2 & témoigner \\
\hline
\end{tabular}




\begin{tabular}{|c|c|c|}
\hline tester & 2 & bavarder \\
\hline tonner & 2 & bedonner \\
\hline traiter & 2 & besogner \\
\hline trottiner & 2 & bifurquer \\
\hline veiller & 2 & blesser \\
\hline venter & 2 & boiter \\
\hline virer & 2 & bouillir \\
\hline voler & 2 & bouler \\
\hline abaisser & 1 & boulotter \\
\hline abandonner & 1 & bousculer \\
\hline accepter & 1 & boxer \\
\hline accoler & 1 & braire \\
\hline accompagner & 1 & brasser \\
\hline accourir & 1 & broder \\
\hline accroître & 1 & bronzer \\
\hline accueillir & 1 & broyer \\
\hline acesmer & 1 & bûcher \\
\hline acheminer & 1 & buter \\
\hline achever & 1 & cabrioler \\
\hline aconter & 1 & cacher \\
\hline acostumer & 1 & caler \\
\hline acoudre & 1 & calmer \\
\hline adestrer & 1 & caracoler \\
\hline adresser & 1 & carapater \\
\hline affonder & 1 & carguer \\
\hline ajourner & 1 & casquer \\
\hline alever & 1 & casser \\
\hline allier & 1 & cataloguer \\
\hline amarrer & 1 & ceindre \\
\hline ambler & 1 & cesser \\
\hline amener & 1 & chaloir \\
\hline apercevoir & 1 & charrier \\
\hline appareiller & 1 & cheviller \\
\hline apporter & 1 & chiqueter \\
\hline apprécier & 1 & choisir \\
\hline areer & 1 & chuchoter \\
\hline arer & 1 & circuler \\
\hline arraisonner & 1 & ciseler \\
\hline arroyer (areer) & 1 & citer \\
\hline articuler & 1 & clapoter \\
\hline aspirer & 1 & clouer \\
\hline assommer & 1 & cogiter \\
\hline atteindre & 1 & coller \\
\hline avancer & 1 & colloquer \\
\hline avouer & 1 & colorer \\
\hline baer & 1 & comparer \\
\hline barboter & 1 & composer \\
\hline barder & 1 & conclure \\
\hline barrer & 1 & condamner \\
\hline
\end{tabular}

\begin{tabular}{|c|c|c|c|}
\hline 1 & confier & 1 & devoir \\
\hline 1 & confondre & 1 & diagnostiquer \\
\hline & conforter & 1 & dîner \\
\hline & conjecturer & 1 & discuter \\
\hline & conjouir & 1 & ébattre \\
\hline & conreer & 1 & échapper \\
\hline & consommer & 1 & éclore \\
\hline & contenir & 1 & écorcher \\
\hline & correspondre & 1 & écraser \\
\hline & corseter & 1 & écumer \\
\hline & cosser & 1 & effacer \\
\hline & coter & 1 & embarrer \\
\hline & coudre / acoudre & 1 & embêter \\
\hline & courroucer & 1 & emblaver \\
\hline & couver & 1 & émouver \\
\hline & craindre & 1 & emparler \\
\hline & crêper & 1 & emprisonner \\
\hline & creuser & 1 & encager \\
\hline & dater & 1 & encaisser \\
\hline & décapiter & 1 & encontrer \\
\hline & déchaumer & 1 & enformer \\
\hline & déchevaucher & 1 & enfouir \\
\hline & déchirer & 1 & engueuler \\
\hline & décider & 1 & enlacer \\
\hline & décocher & 1 & ennouer \\
\hline & décoincer & 1 & entamer \\
\hline & décoller & 1 & enterrer \\
\hline & décolleter & 1 & entreheurter \\
\hline & décorer & 1 & entrelacer \\
\hline & découper & 1 & entre-pousser \\
\hline & décrire & 1 & éplucher \\
\hline & déduire & 1 & épurger \\
\hline & déguster & 1 & équiper \\
\hline & démarrer & 1 & esbanier \\
\hline & départir & 1 & essouffler \\
\hline & dépenser & 1 & étinceler \\
\hline & déplier & 1 & étoiler \\
\hline & déployer & 1 & étouffer \\
\hline . & déporter & 1 & éveiller \\
\hline 1 & dépouiller & 1 & examiner \\
\hline 1 & dérouler & 1 & exciter \\
\hline 1 & désafeutrer & 1 & expliquer \\
\hline & désarêter & 1 & façonner \\
\hline & désarmer & 1 & faucher \\
\hline & déshydrater & 1 & fêler \\
\hline & destiner & 1 & ferrer \\
\hline & détourner & 1 & figer \\
\hline & dévaler & 1 & figurer \\
\hline & deviner & 1 & finer \\
\hline
\end{tabular}




\begin{tabular}{|c|c|c|c|c|c|c|}
\hline fleurir & 1 & mêler & 1 & prenez & 1 & retraire \\
\hline fonctionner & 1 & ménager & 1 & pressentir & 1 & retravailler \\
\hline fouetter & 1 & mendier & 1 & prévenir & 1 & retrouver \\
\hline fourrer & 1 & meugler & 1 & prévoir & 1 & rêvasser \\
\hline fraîchir & 1 & mouvoir & 1 & promener & 1 & reverser \\
\hline fredonner & 1 & murer & 1 & protester & 1 & revertir \\
\hline freiner & 1 & muser & 1 & quitter & 1 & rhétoriquer \\
\hline frotter & 1 & nager & 1 & rabattre & 1 & ricaner \\
\hline gaffer & 1 & neiger & 1 & racheter & 1 & rimer \\
\hline galoper & 1 & nourrir & 1 & ramener & 1 & risquer \\
\hline garnir & 1 & nuire & 1 & ramper & 1 & ronfler \\
\hline gaufrer & 1 & obtenir & 1 & râper & 1 & rouer \\
\hline gerber & 1 & œuvrer & 1 & ravaler & 1 & sangler \\
\hline germer & 1 & oublier & 1 & réagir & 1 & sculpter \\
\hline gesticuler & 1 & paraître & 1 & réallumer & 1 & séjourner \\
\hline goder & 1 & parer & 1 & réarmer & 1 & sigler \\
\hline goter & 1 & paumoier & 1 & rebondir & 1 & signaler \\
\hline gouverner & 1 & paver & 1 & rebouter & 1 & souffrir \\
\hline gracier & 1 & peigner & 1 & rebrousser & 1 & souhaiter \\
\hline griller & 1 & peiner & 1 & rechausser & 1 & soupçonner \\
\hline guimpler & 1 & peinturlurer & 1 & récrier & 1 & stopper \\
\hline habiter & 1 & pelotonner & 1 & recueillir & 1 & suivre \\
\hline hâler & 1 & pénétrer & 1 & reculer & 1 & supposer \\
\hline halluciner & 1 & percevoir & 1 & redescendre & 1 & surveiller \\
\hline hâter & 1 & percuter & 1 & redraper & 1 & swinguer \\
\hline hennir & 1 & perler & 1 & redresser & 1 & taire \\
\hline honnir & 1 & perruqué & 1 & referir & 1 & talocher \\
\hline imaginer & 1 & perturber & 1 & refermer & 1 & tapisser \\
\hline importer & 1 & piaffer & 1 & réfléchir & 1 & tartiner \\
\hline imprimer & 1 & picoler & 1 & rejoindre & 1 & temprer (tremper) \\
\hline incarner & 1 & piler & 1 & réjouir & 1 & terminer \\
\hline incendier & 1 & piller & 1 & relayer & 1 & tisser \\
\hline informer & 1 & pilonner & 1 & relever & 1 & tordre \\
\hline interrompre & 1 & piqueniquer & 1 & reluire & 1 & tornoyer \\
\hline jacasser & 1 & plaire & 1 & remettre & 1 & tourner (atourner) \\
\hline jucher & 1 & plais(s)ier & 1 & remiser & 1 & traîner \\
\hline lamenter & 1 & planer & 1 & remonter & 1 & transpirer \\
\hline laquer & 1 & player & 1 & rentrer & 1 & transporter \\
\hline larder & 1 & plier & 1 & renvoyer & 1 & traverser \\
\hline lever & 1 & plonger & 1 & repairer & 1 & trébucher \\
\hline losangier & 1 & ponter & 1 & repentir & 1 & trépasser \\
\hline louvoyer & 1 & poudrer & 1 & reposer & 1 & tressauter \\
\hline mâcher & 1 & pourpenser & 1 & resplendir & & tresser \\
\hline mailler & 1 & poursuivre & 1 & (resplendre) & 1 & trimer \\
\hline marier & 1 & pourvoir & 1 & ressentir & 1 & triquer \\
\hline marmotter & 1 & pratiquer & 1 & resserrer & 1 & tromper \\
\hline marquer & 1 & prêcher & 1 & retirer & 1 & trompeter (tromper) \\
\hline mastiquer & 1 & précipiter & 1 & retomber & 1 & trompeter \\
\hline médire & 1 & prédire & 1 & retordre & 1 & troubler \\
\hline
\end{tabular}




$\begin{array}{llllll}\text { trouver } & 1 & \text { varier } & 1 & \text { voiler } & 1 \\ \text { user } & 1 & \text { vesser } & 1 & \text { vomir } & 1 \\ \text { vanter } & 1 & \text { virevolter } & 1 & \text { voyager } & 1\end{array}$


